\title{
Abnormal Ultraviolet Mutagenic Spectrum in Plasmid DNA Replicated in Cultured Fibroblasts from a Patient with the Skin Cancer-prone Disease, Xeroderma Pigmentosum
}

Saraswathy Seetharam, ${ }^{\star}$ Miroslava Protić-Sabljić,` Michael M. Seidman, ${ }^{\star}$ and Kenneth H. Kraemer

*Laboratory of Molecular Carcinogenesis, National Cancer Institute, Bethesda, Maryland 20892;

${ }^{\ddagger}$ Otsuka Pharmaceutical Company; Rockville, Maryland 20850

\begin{abstract}
A shuttle vector plasmid, pZ189, was utilized to assess the types of mutations that cells from a patient with xeroderma pigmentosum, complementation group $\mathrm{D}$, introduce into ultraviolet (UV) damaged, replicating DNA. Patients with xeroderma pigmentosum have clinical and cellular UV hypersensitivity, increased frequency of sun-induced skin cancer, and deficient DNA repair. In comparison to UV-treated pZ189 replicated in DNA repair-proficient cells, there were fewer surviving plasmids, a higher frequency of plasmids with mutations, fewer plasmids with two or more mutations in the marker gene, and a new mutagenic hotspot. The major type of base substitution mutation was the $G: C$ to $A: T$ transition with both cell lines. These results, together with similar findings published earlier with cells from a xeroderma pigmentosum patient in complementation group A, suggest that isolated G:C to A:T somatic mutations may be particularly important in generation of human skin cancer by $\mathrm{UV}$ radiation.
\end{abstract}

\section{Introduction}

Xeroderma pigmentosum is a rare, autosomal recessive disease predisposing affected individuals to excessive sunlightinduced skin damage and a $>1,000$-fold increased frequency of skin cancer (1-5). Cells from most xeroderma pigmentosum patients are hypersensitive to the lethal and mutagenic effects of ultraviolet (UV) radiation and are deficient in the ability to repair UV-induced DNA damage (3-6). Base substitution mutations in somatic cells have been shown to be capable of activating certain proto-oncogenes (7). The finding of cellular UV hypermutability makes xeroderma pigmentosum one of the strongest supports for the thesis that somatic mutations play a role in induction of skin cancers in humans.

There are presently identified nine complementation groups among excision repair-deficient xeroderma pigmentosum cells $(3,4)$. Each complementation group generally has a characteristic rate of residual DNA repair (3-5) and kinetics of complementation (6), implying that they carry mutations at different loci. All are believed to involve defects in the first

Dr. Protić-Sabljić's present address is Laboratory of Marine Molecular Biology, Rudjer Bošković Institute, Zagreb, Yugoslavia. Address correspondence to Dr. Kraemer, National Cancer Institute, Laboratory of Molecular Carcinogenesis, Building 37, Room 3E24, Bethesda, MD 20892.

Received for publication 26 January 1987 and in revised form 26 June 1987.

The Journal of Clinical Investigation, Inc.

Volume 80, December 1987, 1613-1617 (incision) step of excision repair but in none are the molecular details understood. Most are associated with increased frequency of skin cancer (1-5). Mutation data from xeroderma pigmentosum cells in different complementation groups would be relevant to understanding the role of mutagenesis in the activation of proto-oncogenes.

We previously utilized a mutagenesis shuttle vector plasmid (8), pZ189, containing a small marker gene (supF coding for a tyrosine suppressor transfer RNA (tRNA), 160 basepairs) to measure mutagenesis in cells from a patient (9) with xeroderma pigmentosum complementation group A (XP-A) ${ }^{1}$ who had multiple nonmelanoma skin cancers and moderate neurological degeneration. The UV mutation spectrum was markedly restricted with the XP-A cells in comparison with a normal line (8). In the present study we examined the mutagenic spectrum of pZ189 passaged through cells of a patient with xeroderma pigmentosum complementation group D (XP-D) who had melanoma and nonmelanoma skin cancers and severe neurological degeneration. Clinical manifestations of this syndrome might be the result of the hypermutability of XP-D cells due to a reduced ability to perform error-free repair of DNA damage.

\section{Methods}

Patient. An SV40 immortalized skin fibroblast line, XP6BE(SV40), XP-D, was established (10) from a 20 year old female with xeroderma pigmentosum, complementation group D (9). (This line, designated GM8207, is available from the Human Genetic Mutant Cell Repository, Camden, NJ.) The patient had more than 25 skin cancers including basal cell carcinomas, squamous cell carcinomas, and two superficial spreading malignant melanomas. She had mental retardation (IQ 49), areflexia, and sensorineural deafness (9). She died at $29 \mathrm{yr}$ of age of metastatic cancer.

Methods. The shuttle vector mutagenesis assay with plasmid pZ189 was performed as previously described $(8,11)$. Briefly, the plasmid was treated with $254 \mathrm{~nm} \mathrm{UV} \mathrm{radiation}\left(0,100,300\right.$, or $\left.500 \mathrm{~J} \cdot \mathrm{m}^{-2}\right)$ from a germicidal lamp, and transfected into the XP-D cells by a calcium phosphate precipitation procedure. Plasmid repair, replication, and mutation utilizing the host cell enzymes was permitted to proceed for 2 $\mathrm{d}$ and resulted in maximal plasmid yield. Since photoproducts are not present in newly synthesized DNA, UV-induced mutations must of necessity occur during the first few cycles of replication in the human cells. Subsequent replication only expands the size of the mutant population of plasmids. The (episomal) plasmids were extracted by the method of Hirt and treated with Dpn I to eliminate the input plasmid that has methylated adenines typical of replication in bacteria. The low molecular weight DNA was purified and introduced into competent Escherichia coli strain MBM7070 cells, which were spread on LB agar

1. Abbreviations used in this paper: TT, thymine-thymine; XP-A and XP-D, xeroderma pigmentosum complementation groups A and D. 
plates containing ampicillin $(50 \mu \mathrm{g} / \mathrm{ml})$, the indicator dye, $X$-gal, and the inducer of beta galactosidase, 5 -bromo-4-chloro-3-indolyl $\beta$-D-galactoside (X-Gal), and isopropyl $\beta$-D-thiogalactoside (IPTG).

Bacteria expressing the plasmid become ampicillin resistant. Thus, the number of colonies growing on the ampicillin-containing plates reflects plasmid survival. The indicator strain of bacteria contains a suppressible (amber) mutation in the lac $Z$ gene which codes for beta galactosidase. Plasmids with an unmutated marker sup $F$ tRNA gene will suppress the mutation in the lac $Z$ gene, thereby resulting in synthesis of beta galactosidase. This enzyme will metabolize the colorless $\mathrm{X}$-gal in the agar and result in a blue colony. On the other hand, if the supF gene sustains a mutation that inactivates its expression, it will not suppress the lac $Z$ mutation and the colony will be white. Partial inactivation of the lac $Z$ gene results in a light blue colony. Cell survival is unaffected by the status of the supF gene. Single or tandem base substitution mutations at $54 \%$ of the basepairs ( 75 of 139 basepairs from basepair 42 to basepair 180 ) have been found to reduce expression of the supF gene $(8,11-13$, and our unpublished observations). No silent mutations have yet been identified. The proportion of white or light blue colonies thus reflects the mutation frequency of the supF gene in the replicated plasmid. Mutant plasmids were purified and doublestranded DNA sequencing was performed with a dideoxy technique.

\section{Results}

The plasmid was treated with different doses of UV radiation and propagated in the XP-D cells. $2 \mathrm{~d}$ later the replicated plasmids were purified and used to transform indicator bacteria. UV treatment of pZ189 decreased its survival to a greater extent with the XP-D cells than with the repair-proficient human cells (GM0637 [SV40]) (Fig. 1 $A$ ). The spontaneous mutation frequency of the supF gene in pZ189 propagated through XP-D cells was $0.06 \%$, a frequency similar to that obtained with the repair-proficient cells (8). The frequency of plasmids with mutations after UV treatment increased in a dose-dependent manner with both cells lines. However, there was a greater increase in mutation frequency with the XP-D line so that treatment of $\mathrm{pZ1} 189$ with $500 \mathrm{~J} \cdot \mathrm{m}^{-2}$ resulted in sixfold higher mutation frequency than with the normal line (Fig. 1 B).

Sequence analysis of 69 mutated plasmids of wild type size recovered from passage through the XP-D cells showed all to be point mutations with no small insertions or deletions (Table I). The point mutations consisted of single base substitutions, as well as tandem and multiple base substitutions in individual mutant plasmids. Table I compares these data with that previously obtained with an XP-A line and a repair-proficient human line $(8)$. There were significantly fewer $(P<0.05)$ tandem base substitution mutations with UV-treated pZ189 propagated in XP-D cells than in the repair-proficient human (8) (or monkey kidney [11]) cell lines or in the XP-A cell line previously tested $(8)$. There was also a significant $(P<0.01)$ reduction in frequency of plasmids with multiple base substitution mutations (and a corresponding significant $[P<0.01]$ increase in single base substitution mutations) with both xeroderma pigmentosum lines in comparison with the results with the repair-proficient human cells.

In Table II the types of base substitution mutations observed in UV-treated pZ189 passaged through the XP-D cell line are compared with those previously found (8) in the XP-A line and in the repair-proficient human line. With all three cell lines the major class of single or tandem base substitution mutations found was the G:C to A:T transition, and only
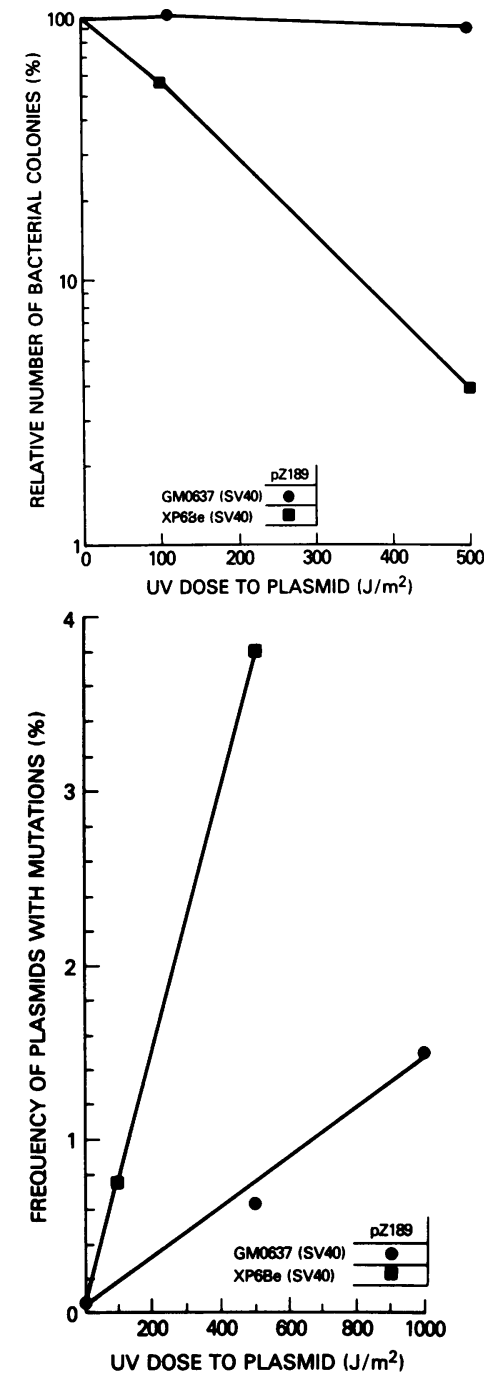

Figure 1. Survival and mutations in UV-treated pZ189 replicated in the XP-D cell line, XP6BE(SV40). (A) Relative number of ampicillin-resistant bacterial colonies obtained after repair and replication of UV-treated pZ189 in XP-D cells (squares) followed by transformation of the indicator bacteria. A representative experiment is shown. Comparable data with the repair-proficient line GM0637(SV40) (circles) can be obtained from reference 8. (B) Frequency ofplasmids with mutations after repair and replication of UV-treated pZ189 in XP-D cells (squares) followed by transformation of the indicator bacteria. A representative experiment is shown. Comparable data with the repair-proficient line GM0637(SV40) (circles) can be obtained from reference 8 .

$5-11 \%$ of the mutations involved A:T basepairs. This is a striking departure from results expected on the basis of UV photoproduct induction studies in pZ189, where the major DNA photoproduct is the thymine-thymine (TT) dimer (12). The plasmid passaged through the XP-D line had significantly $(P<0.05)$ more G:C to T:A transversions than with the XP-A line and significantly $(P<0.05)$ fewer A:T to T:A transversions than with the repair-proficient line (Table II). The frequency of types of mutations with the repair-proficient human line was similar to that reported for a repair-proficient monkey line (11).

The distribution of mutations found in UV-treated pZ189 passaged through the XP-D line was compared with that found with the repair-proficient human line (Fig. 2). The distribution of mutations found in the marker gene was not uniform with either cell line. There were G:C to A:T transition hotspots at basepairs 156 and 123 with both lines (as well as with the XP-A line [8]). With the XP-D line there was a prominent hotspot at basepair 159 , which was significantly $(P<0.001)$ more frequent than with the other lines. Hotspots at basepair 168 were prominent only with the two xeroderma pigmentosum lines (Fig. 2 and reference 8). There were fewer basepairs that were sites of base substitution mutations with XP-D cells than with the repair-proficient line ( 16 vs. $37, P<0.01)$. With XP-D cells all 59 single base substitution mutations were found at sites of 
Table I. Mutations in UV-treated Shuttle Vector PZ189 Replicated in Xeroderma Pigmentosum or Repair-proficient Human Cells

\begin{tabular}{|c|c|c|c|}
\hline & \multicolumn{3}{|c|}{ Number of plasmids with base changes (\%) } \\
\hline & \multicolumn{2}{|c|}{ Xeroderma pigmentosum } & \multirow{2}{*}{$\begin{array}{l}\text { Normal } \\
(\text { GM0637) }\end{array}$} \\
\hline & XP-D* & $X P-A^{\neq 5}$ & \\
\hline \multicolumn{4}{|l|}{ Independent plasmids } \\
\hline sequenced & $69(100)$ & $61(100)$ & $89(100)$ \\
\hline \multicolumn{4}{|l|}{ Point mutations: } \\
\hline Single base substitutions & $59^{\top}(86)$ & $47^{\top}(77)$ & $48(54)$ \\
\hline Tandem base substitutions & $4^{* * \ddagger \ddagger}(6)$ & $12(20)$ & $16(18)$ \\
\hline Multiple base substitutions & $6^{9}(9)$ & $1^{\prime}(2)$ & $24(27)$ \\
\hline \multicolumn{4}{|l|}{ Base insertions and deletions: } \\
\hline Single base insertion & 0 & 0 & $2[2]^{\S \S}$ \\
\hline $\begin{array}{l}\text { Single or tandem base } \\
\text { deletions }\end{array}$ & 0 & $1(2)$ & $3[2]]^{\S}(1)$ \\
\hline
\end{tabular}

* XP6BE (SV40), pZ189 treatment $100-500 \mathrm{~J} \cdot \mathrm{m}^{-2}$.

${ }^{\ddagger} \mathrm{XP12BE}$ (SV40), pZ189 treatment $50-300 \mathrm{~J} \cdot \mathrm{m}^{-2}$.

${ }^{\S}$ Data is from reference 8 .

" pZ189 treatment, $100-1,000 \mathrm{~J} \cdot \mathrm{m}^{-2}$.

' $P<0.01$ vs. GM0637.

** $P<0.05$ vs. GM0637.

\$ $P<0.05$ vs. XP-A.

$\$$ Number of plasmids with insertion or deletion accompanied by one or two base substitutions.

adjacent pyrimidines (i.e., sites of potential UV photoproducts), but only 3 of 67 single or tandem mutations were found at potential TT dimer sites (basepairs 120 and 165). All isolated single base substitution mutations at $\mathrm{G}: \mathrm{C}$ basepairs occurred at a $\mathrm{C}$ that can be interpreted as being $3^{\prime}$ to a $\mathrm{T}$ or $\mathrm{C}$

Table II. Types of Single or Tandem Base Substitutions in UVtreated Shuttle Vector PZ189 Replicated in Xeroderma Pigmentosum or Repair-proficient Human Cells

\begin{tabular}{|c|c|c|c|}
\hline & \multicolumn{3}{|c|}{ Number of changes $(\%)$} \\
\hline & \multicolumn{2}{|c|}{ Xeroderma pigmentosum } & \multirow{2}{*}{$\begin{array}{l}\text { Normal } \\
(\text { GM0637) }\end{array}$} \\
\hline & XP-D* & $X P-A^{\neq 5}$ & \\
\hline Transitions & $59(88)$ & $67^{\top}(94)$ & $61(75)$ \\
\hline $\mathrm{G}: \mathrm{C}$ to $\mathrm{A}: \mathrm{T}$ & $57(85)$ & $66^{9}(93)$ & $59(73)$ \\
\hline A:T to G:C & $2(3)$ & $1(1)$ & $2(2)$ \\
\hline Transversions & $8(12)$ & $4^{\prime}(6)$ & $20(25)$ \\
\hline $\mathrm{G}: \mathrm{C}$ to $\mathrm{T}: \mathrm{A}$ & $5^{* *}(7)$ & $0^{1}$ & $8(10)$ \\
\hline $\mathrm{G}: \mathrm{C}$ to $\mathrm{C}: \mathrm{G}$ & $1(1)$ & $1(1)$ & $5(6)$ \\
\hline $\mathrm{A}: \mathrm{T}$ to $\mathrm{T}: \mathrm{A}$ & $0^{\ddagger \ddagger}$ & $3(4)$ & $6(8)$ \\
\hline$A: T$ to $C: G$ & $2(3)$ & 0 & $1(1)$ \\
\hline Total & $67(100)$ & $71(100)$ & $81(100)$ \\
\hline
\end{tabular}

\footnotetext{
* XP6BE (SV40), pZ189 treatment $100-500 \mathrm{~J} \cdot \mathrm{m}^{-2}$.

$\ddagger$ XP12BE (SV40), pZ189 treatment $50-300 \mathrm{~J} \cdot \mathrm{m}^{-2}$.

${ }^{8} \mathrm{Data}$ is from reference 8 .

" pZ189 treatment $100-1,000 \mathrm{~J} \cdot \mathrm{m}^{-2}$.

$' P<0.01$ vs. GM0637.

** $P<0.05$ vs. XP-A.

¥ $P<0.05$ vs. GM0637.
}

with only one exception (basepair 160). The few tandem mutations with XP-D were all transitions at CC sites.

\section{Discussion}

Plasmid vectors, by demonstrating the action of cellular repair, replication, or mutational systems, may serve as models for understanding the molecular abnormalities in xeroderma pigmentosum. Using a nonreplicating plasmid vector we have previously shown that one pyrimidine dimer is sufficient to inhibit expression of a transfected gene in cells from XP-A and $\mathrm{XP}-\mathrm{D}(14)$. In that assay, which primarily measures differences in transcription, the results with XP-D were similar to those with the XP-A line (XP12BE [SV40]): both showed a 12-fold greater sensitivity to UV than with the repair-proficient cells. In the present study with the replicating plasmid pZ189, survival was also greatly reduced by UV treatment both with the XP-A cells (8) and with XP-D (Fig. $1 A$ ). These results are similar to the relative UV sensitivities of the respective fibroblast strains (3). The hypermutability of pZ189 with SV40transformed XP-D cells (Fig. $1 B$ ) also is similar to that detected with nontransformed XP-D cells after UV treatment of the cells and selection for diphtheria toxin resistance (15) or 6-thioguanine resistance (16).

The mutagenesis plasmid vector enables identification of the types of mutations induced by UV damage in DNA replicating in human cells. The major sites of UV mutagenesis are not at TT sequences, despite their being the major sites of potential UV photoproducts (12). These observations recall those made more than 20 years ago in bacteria $(17,18)$ and discussed recently by several authors $(8,11,12,19,20)$ : There is an apparent "A rule" wherein many prokaryotic and eukaryotic polymerases when encountering noninstructional DNA lesions in the DNA template insert A in the newly synthesized strand. In the case of TT photoproducts the correct nucleotides would be inserted. Photoproducts involving TC or $\mathrm{CC}$ would result in isolated or tandem G:C to A:T transitions, as observed with the XP-D (Fig. 2) and XP-A (8) cells.

The types of mutations observed with the XP-D and XP-A cells are a subset of those seen with the repair-proficient cells. In particular, the multiple base substitution mutations are significantly reduced in frequency with both complementation groups. This suggests that the multiple base substitutions are linked to a normally functioning excision repair system. Indeed, we have recently found that with the XP-A cells, introduction of a single-strand nick in the plasmid (mimicking the first step in excision repair) resulted in a dramatic increase in the frequency of plasmids with multiple base substitution mutations (21).

With the XP-D cells there was also a decrease in frequency of tandem base substitutions, suggesting that generation of this type of mutation is linked to a function that is present in normal and XP-A cells but missing from the XP-D line. Photoreactivation studies indicate that tandem base substitution mutations in pZ189 are primarily caused by cyclobutane pyrimidine dimers $(12,22)$. There is evidence $(23-25)$ that in UV-treated, nontransformed XP-D (and XP-A) fibroblasts there is an accumulation of partially excised modified pyrimidine dimer photoproducts. With XP-D these are accompanied by ineffective repair replication $(24,25)$ that might result in plasmid lethality at potential sites of tandem mutations (Fig. 


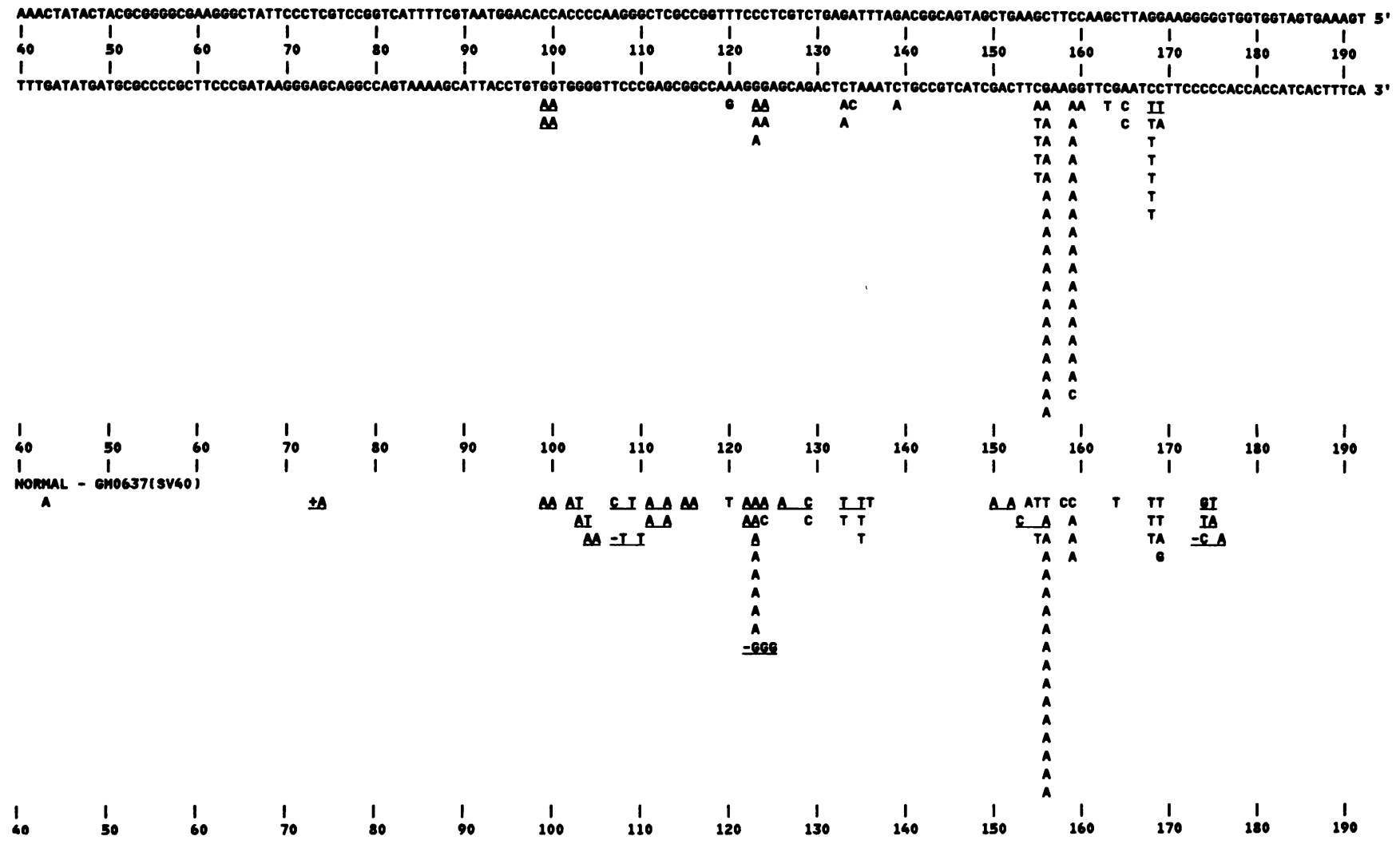

Figure 2. Location of independent single and tandem base substitution mutations found in UV-treated pZ189 replicated in the XP-D cell line, XP6BE(SV40). The portion of the plasmid sequence shown contains the marker gene (supF suppressor tRNA) sequence (basepairs 99 to 183), the pre-tRNA sequence (basepairs 59 to 98), and a portion of the promoter (basepairs 24 to 58). Base substitutions are

2). Partially excised modified photoproducts might be mutagenic, and result in the hotspot at basepair 159 with the XP-D line and at 168 with the XP-A line.

The mutagenic spectrum with the XP-D cells, like that with the XP-A cells (8), is to a first approximation a subset of the mutagenic spectrum with the normal cells. There are fewer basepairs involved in base substitution mutations (Fig. 2) and more single base substitution mutants (Table I) than with the repair-proficient cells. These xeroderma pigmentosum patients had a high frequency of UV-induced skin cancers. Thus, if the shuttle vector plasmid mimics the mutagenic spectrum in the cellular DNA, then somatic mutations leading ultimately to skin cancers are more likely to be those shared by the xeroderma pigmentosum and normal lines: namely, single base substitution transition mutations of the type $\mathrm{G}: \mathrm{C}$ to $\mathrm{A}: \mathrm{T}$.

\section{References}

1. Kraemer, K. H., M. M. Lee, and J. Scotto. 1984. DNA repair protects against cutaneous and internal neoplasia: evidence from xeroderma pigmentosum. Carcinogenesis. 5:511-514.

2. Kraemer, K. H., M. M. Lee, and J. Scotto. 1987. Xeroderma pigmentosum: cutaneous, ocular, and neurologic abnormalities in 830 published cases. Arch. Dermatol. 123:241-250.

3. Kraemer, K. H., and H. Slor. 1985. Xeroderma pigmentosum. Clin. Dermatol. 3:33-69.

4. Cleaver, J. E. 1983. Xeroderma pigmentosum. In The Metabolic

indicated below the altered base pair as a change in the lower strand. Each letter represents the mutation found in a sequenced independent plasmid. Tandem or closely spaced base substitutions, deletions, or insertions in a single plasmid are indicated by underlining. Comparable data for the repair-proficient line GM0637(SV40) can be obtained from reference 8 .

Basis of Inherited Disease. J. B. Stanbury, J. B. Wyngaarden, D. S. Frederickson, J. L. Goldstein, and M. S. Brown, editors. 5th ed. McGraw-Hill, Inc., New York. 1227-1248.

5. Kraemer, K. H. 1987. Heritable diseses with increased sensitivity to cellular injury. In Dermatology in General Medicine. T. B. Fitzpatrick, A. Z. Eisen, K. Wolff, I. M. Freedberg, and K. F. Austen, editors. 3rd ed. McGraw Hill, Inc., New York. 1791-1811.

6. Gianelli, F., S. A. Pawsey, and J. A. Avery. 1982. Differences in patterns of complementation of the more common groups of xeroderma pigmentosum: possible implications. Cell. 29:451-458.

7. Weiss, R. A., and C. J. Marshall. 1984. Oncogenes. Lancet. ii: $1138-1142$.

8. Bredberg, A., K. H. Kraemer, and M. M. Seidman. 1986. Restricted mutational spectrum in an UV-treated shuttle vector propagated in xeroderma pigmentosum cells. Proc. Natl. Acad. Sci. USA. 83:8273-8277.

9. Robbins, J. H., K. H. Kraemer, M. A. Lutzner, B. W. Festoff, and H. W. Coon. 1974. Xeroderma pigmentosum: an inherited disease with sun sensitivity, multiple cutaneous neoplasms and abnormal DNA repair. Ann. Intern. Med. 80:221-248.

10. Protić-Sabljić, M., S. Seetharam, M. M. Seidman, and K. H. Kraemer. 1986. An SV40-transformed xeroderma pigmentosum group D cell line: establishment, ultraviolet sensitivity, transfection efficiency and plasmid mutation induction. Mutat. Res. 166:287-294.

11. Hauser, J., M. M. Seidman, K. Sidur, and K. Dixon. 1986. Sequence specificity of point mutations induced during passage of a UV-irradiated shuttle vector plasmid in monkey cells. Mol. Cell. Biol. 6:277-285.

12. Brash, D. F., S. Seetharam, K. H. Kraemer, M. M. Seidman, and A. Bredberg. 1987. Photoproduct frequency is not the major deter- 
minant of UV base substitution hot spots or cold spots in human cells. Proc. Natl. Acad. Sci. USA. 84:3782-3786.

13. Yang, J.-L., V. M. Maher, and J. J. McCormick. 1987. Kinds of mutations formed when a shuttle vector containing adducts of $( \pm)-7$ beta, 8 alpha-dihydroxy-9 alpha, 10 alpha-epoxy-7,8,9,10tetrahydrobenzo[a]pyrene replicates in human cells. Proc. Natl. Acad. Sci. USA. 84:3787-3791.

14. Protić-Sabljić, M., and K. H. Kraemer. 1985. One pyrimidine dimer inactivates expression of a transfected gene in xeroderma pigmentosum cells. Proc. Natl. Acad. Sci. USA. 82:6622-6626.

15. Glover, T. W., C. C. Chang, J. E. Trosko, and S. S. Li. 1979. Ultraviolet light induction of diphtheria-toxin resistant mutants of normal and xeroderma pigmentosum human fibroblasts. Proc. Natl. Acad. Sci. USA. 76:3982-3986.

16. Simons, J. W. I. M. 1979. Development of a liquid holding technique for the study of DNA repair in human diploid fibroblasts. Mutat. Res. 59:273-283.

17. Drake, J. W. 1963. Properties of ultraviolet-induced rII mutants of bacteriophage T4. J. Mol. Biol. 6:268-283.

18. Howard, B. D., and I. Tessman. 1964. Identification of the altered bases in mutated single-stranded DNA III. Mutagenesis by ultraviolet light. J. Mol. Biol. 9:372-375.

19. Strauss, B. S., S. Rabkin, S. Sagher, and P. Moore. 1982. The role of DNA polymerase in base substitution mutagenesis on non-instructional templates. Biochimie. 64:829-838.
20. Schaaper, R. M., T. A. Kunkel, and L. Loeb. 1983. Infidelity of DNA synthesis associated with bypass of apurinic sites. Proc. Natl. Acad. Sci. USA. 80:487-491.

21. Seidman, M. M., A. Bredberg, S. Seetharam, and K. H. Kraemer. 1987. Multiple point mutations in a shuttle vector propagated in human cells: evidence for an error-prone DNA polymerase activity. Proc. Natl. Acad. Sci. USA. 84:4944-4948.

22. Protić-Sabljić, M., N. Tuteja, P. Munsen, J. Hauser, K. H. Kraemer, and K. Dixon. 1986. UV-induced cyclobutane dimers are mutagenic in mammalian cells. Mol. Cell. Biol. 6:3349-3356.

23. Weinfeld, M., N. Gentner, L. D. Johnson, and M. C. Paterson. 1986. Photoreversal-dependent release of thymidine and thymidine monophosphate from pyrimidine-dimer containing DNA excsion fragments isolated from ultraviolet-damaged human fibroblasts. Biochemistry. 25:2656-2664.

24. Paterson, M. C., N. E. Gentner, M. V. Middlestadt, and M. Weinfeld. 1984. Cancer predispostion, carcinogen hypersensitivity, and aberrant DNA metabolism. J. Cell. Physiol. 3:45-62.

25. Paterson, M. C., N. E. Gentner, M. V. Middlestadt, R. Mirzayans, and M. Weinfeld. 1985. Hereditary and familial disorders linking cancer proneness with abnormal carcinogen response and faulty DNA metabolism. In Epidemiology and Quantitation of Environmental Risk in Humans from Radiation and Other Agents. A. Castellani, editor. Plenum Publishing Corp., New York. 235-267. 